\title{
\begin{tabular}{l|l} 
POLITIQUES \& & Politiques et management public
\end{tabular}

\section{Réglementer la qualité de l'eau potable au Québec : une analyse politico-administrative}

The regulation of drinking water in Quebec: a political and administrative analysis

\section{David Talbot et Jean Turgeon}

\section{(2) OpenEdition}

\section{Journals}

Édition électronique

URL : http://journals.openedition.org/pmp/2964

DOI : $10.4000 / p m p .2964$

ISSN : 2119-4831

Éditeur

Institut de Management Public (IDPM)

Édition imprimée

Date de publication : 15 septembre 2010

Pagination : 5-24

ISSN : 0758-1726

Référence électronique

David Talbot et Jean Turgeon, « Réglementer la qualité de l'eau potable au Québec : une analyse politico-administrative », Politiques et management public [En ligne], Vol 27/4 | 2010, mis en ligne le 15 septembre 2012, consulté le 01 mai 2019. URL : http://journals.openedition.org/pmp/2964 ; DOI : 10.4000/pmp.2964 


\title{
RÉGLEMENTER LA QUALITÉ DE L'EAU POTABLE AU QUÉBEC : UNE ANALYSE POLITICO-ADMINISTRATIVE
}

\author{
David TALBOT*, Jean TURGEON**
}

Résumé

Mots-clés

Abstract

Keywords
Cet article vise la compréhension du contexte et du jeu des acteurs ayant mené à l'adoption du Règlement sur la qualité de l'eau potable. Cette initiative a permis au Québec de se positionner parmi les États ayant des normes de qualité et des contrôles de production parmi les plus rigoureux sur le continent nord-américain. Une adaptation de l'approche contextualiste de Pettigrew (1990; 1992) est retenue comme cadre d'analyse. Cette approche insiste sur l'importance, au moment d'un changement, de l'interaction entre trois dimensions : le contexte, le contenu et le processus. De manière à préciser cette dernière dimension, il est proposé de recourir à l'idée de coalitions, un concept propre à l'approche des réseaux de politiques publiques. Cet apport permet de mieux cerner l'influence des organisations externes à l'appareil gouvernemental et de fournir une explication des relations entre les différentes dimensions du contextualiste.

Gestion de l'eau, approche contextualiste, réseaux de politique, coalitions de cause.

\section{THE REGULATION OF DRINKING WATER IN QUEBEC : A POLITICAL AND ADMINISTRATIVE ANALYSIS}

The objective of this article is to achieve a better understanding of the context and interplay of actors involved in the adoption of Quebec's Regulation respecting the quality of drinking water. This adoption ranks Quebec among those governments of North America having the most stringent standards of quality and production control. As analytical framework of this article, Pettigrew's contextualist approach (1990; 1992) was adapted. This approach emphasizes the importance, at the time of change, of the interaction among three dimensions: the context, the content of change and change processes. With a view to fleshing out the process dimension, this article relies on the notion of advocacy coalitions, borrowing from the policy network approach. This contribution provides a basis for better assessing the influence of external organizations to the government and for explaining relations among different dimensions of the contextualist approach.

Water management, contextualist approach, public policy networks, advocacy coalitions.

\footnotetext{
*Étudiant au doctorat - Faculté des sciences de l'administration, Université Laval, Québec, Canada david.talbot@fsa.ulaval.ca

${ }^{* *}$ Professeur titulaire, responsable et chercheur principal - Groupe d'étude sur les politiques publiques et la santé (GÉPPS) - École nationale d'administration publique, Québec, Canada

jean.turgeon@enap.ca
} 
Introduction

Depuis plus d'un siècle, d'importants investissements ont été consentis par plusieurs pays dans le développement des procédés de traitement et d'analyse de l'eau destinée à la consommation humaine. Au Canada et dans la majorité des pays industrialisés, la filtration et la chloration ont d'ailleurs permis de révolutionner le domaine de l'hygiène publique (Leclerc, 2003; Schoenen, 2002). Malgré ces efforts, les épidémies d'origine hydrique demeurent une préoccupation de santé publique dans les pays développés (Levallois, 2006). Aux États-Unis, de 1991 à 2002, 207 éclosions de maladies reliées à la consommation d'eau ont été observées et 73 décès liés à ces éclosions ont été rapportés (Craun, 2006). Au Canada, une situation similaire est également observée puisque 288 épidémies reliées à l'eau potable ont eu lieu entre 1974 et 2001 (Schuster et al., 2005). L'événement le plus dramatique de cette période est survenu en mai 2000, dans la ville de Walkerton en Ontario. La contamination par la bactérie E. coli de l'eau distribuée a causé la mort de six personnes en plus des 1346 cas déclarés de gastro-entérite (Hrudey et al., 2003). Afin d'assurer à la population une eau sécuritaire, la majorité des provinces canadiennes et plusieurs pays ont adopté des réglementations pour encadrer la distribution de cette ressource (Talbot et Turgeon, 2007).

C'est en 1984 que le Québec est devenu la première province canadienne à se doter d'un règlement intégrant des normes de qualité et des exigences de contrôle de l'eau destinée à la consommation humaine. Cependant, dès 1986 l'idée de modifier ce règlement est à l'agenda du ministère de l'Environnement. Par la suite, au début des années 1990 certaines lacunes au niveau du contrôle (fréquence d'échantillonnage et réseaux assujettis) et de prévention (normes) avaient été identifiées par les autorités gouvernementales (Gouvernement du Québec, 1993). L'encadrement législatif ne permettait pas alors d'assurer les objectifs eu égard à la protection de la santé publique, et ce, particulièrement pour les réseaux desservants moins de 5000 habitants (Ministère de l'Environnement du Québec, 1989). Après 15 années de tergiversations politiques, une nouvelle réglementation est finalement adoptée en mai 2001.

Cet article vise à mieux comprendre l'adoption de ce changement stratégique au sein du ministère de l'Environnement du Québec ${ }^{1}$. Plus spécifiquement, il propose d'identifier puis d'analyser l'influence du contexte et du jeu des acteurs lors de la formulation du règlement québécois sur la qualité de l'eau potable. Afin de réaliser cet objectif, une adaptation de l'approche contextualiste de Pettigrew $(1990 ; 1992)$ est proposée. Ce

\footnotetext{
${ }^{1} \mathrm{Ce}$ ministère a changé d'appellation cinq fois au cours des vingt dernières années : ministère de l'Environnement du Québec, avant 1994; ministère de l'Environnement et de la Faune, de 1994 à 1998; ministère de l'Environnement, de 1998 à 2005; ministère du Développement durable, durant quelques mois en 2005 puis ministère du Développement durable, de l'Environnement et des Parcs. Pour faciliter la lecture le terme ministère de l'Environnement est retenu, peu importe la période.
} 
cadre d'analyse s'intéresse à l'interaction entre trois dimensions pour expliquer un changement stratégique ou organisationnel : le contexte, le contenu et le processus. Depuis la fin des années 1980, peu d'études ont utilisé les approches politiques comme le contextualisme pour l'étude du changement (Demers, 2007). Des recherches sont notamment nécessaires dans le secteur de la santé où les travaux ont majoritairement porté sur l'implantation de systèmes d'information (Cho et al., 2008 ; Jayasuriya, 1999 ; Pettigrew et al., 1992). De plus, ce cadre est peu utilisé pour étudier des changements d'orientation au niveau des politiques publiques (Skirstad, 2009), l'une de ses limites étant qu'il sous-estime l'importance du rôle des parties prenantes externes à l'organisation dans le processus de changement. Afin de palier à cette lacune et de prendre en considération le jeu de ces acteurs, cet article intègre à son cadre d'analyse l'approche des réseaux de politique publique et plus précisément le concept de coalitions (Sabatier et Jenkins-Smith, 1993 ; 1999). Cette recherche répond ainsi à l'invitation de Macdonald (2001) de continuer à approfondir notre compréhension des jeux de pouvoir dans les réseaux de politique.

Dans un premier temps, l'article présente l'approche contextualiste et celle des réseaux de politiques afin de mettre en exergue la complémentarité de ces théories pour étudier un changement stratégique. Par la suite, la stratégie de recherche sera exposée, de même que les méthodes qui ont été mobilisées pour « reconstruire » le processus décisionnel ayant mené à l'adoption de la réglementation de l'eau potable au Québec. Suivra la description de l'évolution de ce cas puis son analyse au moyen du cadre retenu.

\section{Cadre conceptuel}

\section{L'Approche contextualiste}

À partir d'une revue de la documentation abordant les changements organisationnels, Pettigrew $(1990 ; 1992)$ a constaté que la majorité des études ne prennent pas en considération l'histoire et le processus de transformation. Pour remédier à cette lacune, il propose une nouvelle démarche heuristique visant à expliquer les mécanismes et les processus à travers lesquels un changement voit le jour (Pettigrew et al., 1992). L'approche contextualiste met en relation trois dimensions ayant un lien d'interdépendance (Pettigrew et al., 1992). La première, le contenu (Quoi), réfère aux domaines concernés par les transformations. II s'attarde à expliquer ce qui a été modifié. La deuxième dimension concerne l'environnement interne et externe. Le « contexte externe » correspond à l'environnement social, économique, politique dans lequel se développe et évolue l'organisation. Le « contexte interne » aborde la structure, la culture et la configuration des pouvoirs propres à l'organisation. II influence particulièrement le processus par lequel les idées concernant les changements sont véhiculées. Le troisième élément du cadre d'analyse est le processus de changement. Ce dernier fait référence aux actions, réactions et interactions entre les différents acteurs pour faire passer l'organisation d'un état présent à un état futur. C'est le jeu de force entre les acteurs et la quête de pouvoir qui sont au centre de cet élément. 
L'un des grands défis que pose l'utilisation de ce cadre sur le plan analytique est de pouvoir lier ces trois dimensions à travers le temps (Pettigrew, 1990). L'approche contextualiste se distingue par l'idée de l'interrelation entre le contexte et le processus (Pettigrew et al., 2001). Ainsi, les acteurs sont influencés par l'environnement, mais ils peuvent également l'utiliser pour donner de la légitimité au changement ou pour délégitimiser la position de leurs opposants. Le contenu du changement est le produit du processus de légitimation qui, lui, est influencé par des considérations culturelles et politiques (Pettigrew et al., 1992 ; Pettigrew, 1990). Les événements peuvent être interprétés différemment par les acteurs selon leurs croyances et leurs perceptions. Dans cette perspective, les contextes ne sont pas inertes et objectifs. Pettigrew et ses collègues (1992) ont d'ailleurs identifié, dans le secteur de la santé, huit facteurs interreliés pouvant expliquer la réceptivité de certains contextes aux changements. Ils ont ainsi reconnu l'influence des pressions environnementales, des réseaux interorganisationnels coopératifs, des acteurs clés dans la conduite du changement, des relations entre les gestionnaires et les employés, de la qualité et de la cohérence des politiques, de la simplicité et la clarté des objectifs, de la culture organisationnelle et enfin de la planification du changement. Peu d'études empiriques ont été menées sur la réceptivité des contextes (Caza, 2000 ; Cho et al., 2008 ; Marchionni et Ritchie, 2008). Certains de ces facteurs ont été retenus dans l'étude.

L'article applique l'approche contextualiste à la formulation de la réglementation de l'eau potable. À l'instar de Thibault et Babiak (2005), le changement à l'étude se situe au niveau d'un ensemble d'organisations opérant dans un secteur particulier d'intervention plutôt que dans une seule organisation. La gestion et la distribution de l'eau potable est dépendante d'un grand nombre d'acteurs publics et privés, d'où la pertinence de s'intéresser aux interactions entre les différentes parties prenantes. Cette orientation se démarque des travaux de Pettigrew et ses collaborateurs (1990 ; 1992) qui s'intéressent davantage aux rôles des hauts-dirigeants. Starkey (1987) a d'ailleurs critiqué les travaux fondateurs de Pettigrew en indiquant que les changements stratégiques ne sont pas liés exclusivement au sommet stratégique. L'étude de Thibault et Badiak (2005) a démontré l'apport de l'étude des interactions entre les différentes parties prenantes sur la compréhension du processus et du contexte de changement.

Cependant, l'approche contextualiste manque de clarté et de précision quant à la stratégie ou à la méthodologie à adopter afin d'étudier les différentes composantes du modèle (Girginov et Sandanski, 2008). Afin de palier à cette faiblesse, l'approche des réseaux de politiques sera mobilisée pour étudier le processus de changement. Cette perspective insiste sur l'importance des interactions entre les acteurs publics et privés pour expliquer le processus de changement dans un sous-système particulier d'intervention publique (Adam et Kriesi, 2007). À ce sujet, le contextualiste n'exclut pas l'apport potentiel d'une autre approche à la compréhension d'un phénomène aussi complexe que le changement (Girginov et Sandanski, 2008). Certaines études ont d'ailleurs tenté récemment de tels rapprochements, notamment 
avec la théorie de l'acteur système (Cho et al., 2008) et celle de l'État en crise (Girginov et Sandanski, 2008). Cette combinaison de l'approche contextualiste avec celle des réseaux permet d'interpréter le phénomène à l'étude à l'aide de différentes lunettes théoriques.

\section{L'approche des réseaux}

Le concept de réseau de politique est apparu dans la documentation scientifique au cours des années 1970 (Adam et Kriesi, 2007). L'apport fondamental de ces travaux est d'avoir démontré l'influence des groupes d'intérêts dans le processus d'élaboration des politiques. Dans cette perspective, les réseaux peuvent être perçus comme des coalitions. S'inscrivant dans ce courant, Sabatier et Jenkins-Smith (1999) ont développé le cadre d'analyse des coalitions de cause (ACF) pour expliquer le changement dans l'action publique. Au cours de la dernière décennie, l'ACF s'est peu à peu imposée comme l'une des approches les plus influentes dans le débat scientifique international sur les politiques publiques (John, 2003; Kübler et Maillard, 2009). Ses promoteurs envisagent les changements dans l'action gouvernementale sous l'angle de la compétition que se livrent des coalitions d'acteurs, dont chacune défend un ensemble structuré de croyances et de valeurs (Sabatier et Jenkins-Smith $1993 ; 1999)$. L'une des prémisses de l'ACF est que l'unité d'analyse la plus appropriée pour comprendre le processus de changement est le sous-système. Ce dernier désigne le groupe de personnes (membres de groupes de pression, personnalités politiques, fonctionnaires, chercheurs, journalistes) et/ ou d'organisations qui tentent d'influencer la formulation, l'adoption et la mise en œuvre des politiques publiques d'un domaine particulier et qui interagissent régulièrement entre elles sur une période d'une décennie ou plus (Sabatier et Jenkins-Smith, 1999 ; Sabatier et Weible, 2007). La notion de sous-système rend compte de la complexité des enjeux présents dans la société moderne (Sabatier et Weible, 2007).

Même s'ils proviennent d'horizons différents, les acteurs qui forment un sous-système de politiques publiques créent à l'occasion des alliances, d'où l'émergence de " coalitions de cause » (Sabatier et Jenkins-Smith 1993 ; 1999). Ces regroupements d'acteurs font preuve d'un degré non négligeable de coordination à travers le temps (Sabatier et Jenkins-Smith, 1999) et développent, à partir de leurs ressources et de leurs croyances, un ensemble de stratégies pour influencer les autorités gouvernementales. La coalition qui a l'oreille des décideurs et qui parvient à influencer les preneurs de décisions occupe la position de coalition dominante. Même si les acteurs politiques favorisent les compromis et incorporent dans les politiques gouvernementales des éléments suggérés par différentes coalitions, il est généralement possible de déceler dans une politique publique le système de croyances qui l'a inspirée de manière dominante. Nous proposons, à l'instar de Sabatier et de ses collaborateurs, d'accorder une place centrale aux coalitions dans l'étude de la formulation de la réglementation sur la qualité de l'eau potable. L'utilisation du concept de coalition va permettre d'enrichir la notion de processus et de l'associer 
d'une part avec le contexte, d'autre part avec le contenu, ce qui représente le fondement de l'approche contextualiste. La figure 1 présente le cadre d'analyse de notre étude.

Figure 1 - Le cadre d'analyse d'une politique publique

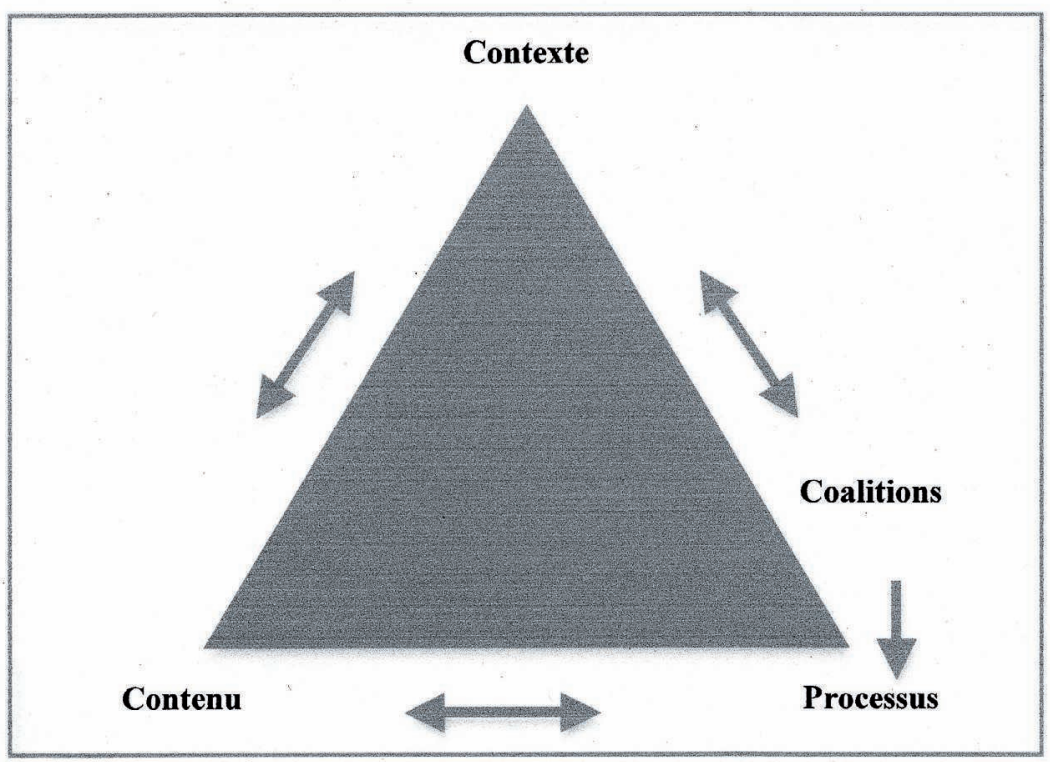

Inspiré de Pettigrew (1987) et Sabatier et Jenkins-Smith (1999)

Afin d'étudier l'influence des coalitions, il est proposé de mobiliser l'analyse structurale des réseaux sociaux. Depuis le début des années 1990, certains chercheurs reconnaissent l'apport potentiel de l'analyse structurale à l'approche des réseaux de politique (Dowding, 2001). Cette stratégie permet d'étudier les interactions entre les acteurs impliqués dans un soussystème d'intervention publique. L'utilisation de ces techniques développées principalement en sociologie tend à s'accroître pour étudier le jeu des acteurs impliqués dans des changements dans l'action gouvernementale (Knoke et al., 1996 ; Kriesi et al., 2006 ; Kriesi et Jegen, 2001 ; Weible et Sabatier, 2005). Pour Weible et Sabatier (2005), c'est l'une des voies de recherche à privilégier pour étudier les relations entre les parties prenantes. Il devient dès lors possible de lier les concepts de coalitions et de processus à une démarche d'opérationnalisation de la notion de réseau.

Méthodologie Dans le cadre de cette étude de cas, une perspective longitudinale a été privilégiée afin de bien comprendre le jeu des acteurs et l'influence des contextes internes et externes sur le processus de changement. La période d'observation débute en 1986 et se termine avec l'adoption de la nouvelle législation en mai 2001. Le choix de l'étude de cas comme stratégie de recherche est appropriée dans le cas présent compte tenu du fait que l'accent est mis sur la compréhension et l'explication d'un phénomène dans 
son contexte réel (Yin, 1994). De plus, l'approche contextualiste privilégie ce type de recherche (Pettigrew et al., 1992).

\section{La collecte et l'analyse des données}

Pour mener à bien cette étude de cas, différents types de données ont été utilisés. Une revue de la littérature grise et de la presse écrite (données secondaires) a été effectuée pour décrire le contenu et le contexte du changement. Par la suite, les mémoires déposés lors de la consultation publique tenue en $2000(n=22)$ ont été analysés afin de mieux comprendre le positionnement et les valeurs des organisations impliquées dans la formulation de cette réglementation. Plus de 48 organisations ont fait parvenir un mémoire. Cependant, aux fins de l'analyse, les organismes locaux ont été exclus. Cette décision a été prise après avoir constaté que la grande majorité de ces mémoires était des lettres modèles signées par les différentes organisations pour appuyer la position de l'organisme national ou régional. Une codification des mémoires a alors été réalisée à partir de six éléments de discorde identifiés par les professionnels du ministère de l'Environnement, soit la fréquence d'échantillonnage, les réseaux touchés, le contrôle des puits individuels, les normes et paramètres, le délai d'application et les programmes d'aide financière. La collecte et l'analyse de ces données secondaires ont été réalisées durant l'automne 2005 par l'un des auteurs. De manière à compléter l'information recueillie lors de l'analyse documentaire, des entretiens semi directifs ont été menées au cours de la même période auprès du gestionnaire et des professionnels responsables du dossier. Les résultats préliminaires de l'étude ont d'ailleurs été présentés lors d'une rencontre avec la direction. Certains des commentaires alors émis ont permis d'enrichir notre compréhension du contexte, du contenu et du processus de changement.

Finalement, un questionnaire a été envoyé à l'automne 2006 aux organisations qui ont été impliquées dans le processus de changement $(n=23)$. Les acteurs retenus devaient répondre à deux critères : (1) avoir participé à la consultation publique ou avoir fait parvenir une lettre au ministère de l'Environnement au cours du processus de formulation et (2) avoir un caractère national ou régional plutôt que local. Le questionnaire utilisé pour repérer les alliances d'acteurs a été développé à partir d'un questionnaire validé dans le cadre d'une étude abordant la protection des espaces marins en Californie (Weible et Sabatier, 2005) qui comprend notamment des informations sur les valeurs et les relations que les acteurs entretiennent dans le cadre de l'élaboration d'une politique. Pour vérifier l'existence de coalitions dans le processus de formulation de la réglementation de l'eau potable, il a été demandé aux différentes organisations d'identifier à partir de la liste des participants $(n=23)$ ceux avec qui elles avaient tenté de coordonner leurs actions pour influencer les décideurs. Le taux de réponse s'établit à un peu plus de $82 \%$ soit, 19 répondants. L'analyse de ces données a été réalisée à l'aide d'une méthode développée par Laumann et ses collaborateurs (Knoke et al., 1996; Laumann et Knoke, 1987; Laumann et Pappi, 1976) pour représenter les réseaux d'acteurs. La stratégie consiste 
à combiner deux méthodes le « hierarchical clustering » avec le « multidimensional scaling ». La première permet d'identifier les regroupements en fonction des équivalences structurelles entre les acteurs tandis que la seconde permet à partir d'une matrice de similarité (ou de dissimilarités) de représenter dans l'espace la distance entre les acteurs du réseau. Dans le cadre de cette étude, le coefficient de similarité de Jaccard a été utilisé. Cette méthode d'analyse a été utilisée par Weible et Sabatier (2005) pour étudier la présence de coalitions de cause. Les différentes analyses ont été réalisées à l'aide du logiciel UCINET (version 6.1).

Résultats

\section{Le contenu et le contexte du changement}

Le changement stratégique à l'étude est le passage d'un règlement axé sur les résultats (1984) à un règlement intégrant des exigences pour les différentes étapes de transformation de l'eau destinée à la consommation humaine (2001). Cette section présente le contenu des trois grandes propositions de règlement $(1993,1998,2000)$ qui ont été étudiées par le ministère de l'Environnement au cours de la période d'observation (19862001). Après avoir présenté chacune des propositions, des liens avec des éléments du contexte interne et externe sont présentés pour expliquer l'évolution du contenu de la proposition.

\section{a. Le projet de 1993}

En 1993, les normes québécoises en vigueur avaient déjà plus de 10 ans et elles étaient contestées depuis le milieu des années 1980. Pour remédier à la situation, un nouveau projet de règlement prévoyant une mise à jour des normes en fonction des nouvelles recommandations canadiennes et internationales a été proposé. Les modifications portaient principalement sur l'aspect bactériologique de la qualité de l'eau. Cette mise à jour provoquait des coûts importants pour les exploitants des systèmes de distribution et pour le ministère de l'Environnement. À l'époque, l'impact économique était évalué à plus de 360 millions de dollars (M\$) (Gouvernement du Québec, 1993). Ce projet a été déposé au Conseil des ministres en mai 1993, cependant les autorités gouvernementales n'y ont pas donné suite, notamment à cause du manque de données épidémiologiques propres au Québec, des coûts potentiels liés à cette modification réglementaire dans un contexte budgétaire difficile et du fait que le ministère de la Santé et des Services sociaux est alors incapable de produire une étude économique jugée valable pour justifier l'intervention gouvernementale dans ce domaine. II faudra attendre en 1998 pour atteindre l'équilibre budgétaire au Québec et, par corolaire, la marge de manœuvre nécessaire pour investir dans de nouveaux programmes d'infrastructures (Charland, 2006).

\section{b. La table d'élaboration et le projet de 1998}

En 1995, une Table d'élaboration est mandatée au sein du ministère de l'Environnement pour étudier différents scénarios. À la suite de ce processus de consultation interne, un nouveau cadre d'intervention réglementaire est 
proposé. II est orienté autour de 5 grands axes de révision : normes de qualité de l'eau, contrôles obligatoires supplémentaires et améliorés de la qualité de l'eau, transfert électronique des données au ministère, actions en cas de dépassements et la désinfection des eaux de surface obligatoire. L'impact économique de cette proposition était minimisé pour le ministère de l'Environnement étant donné que certaines normes comme la turbidité ${ }^{2}$ n'étaient pas mise à jour. Cependant, aucun programme de financement pour les infrastructures n'était prévu pour les petits exploitants privés et publics. Cette proposition avait obtenu l'appui du ministère de la Santé et des Services sociaux, du ministère des Affaires municipales et de l'Association québécoise des techniques de l'eau. Des compromis avaient été consentis pour faciliter la mise à jour du règlement de 1984. Cependant, un événement dans le sous-système a modifié la situation. En 1998, lors de l'ouverture du Congrès annuel de Réseau environnement, Michèle Prévost, de la Chaire en eau potable de l'École polytechnique, et Pierre Payment, de l'Institut ArmandFrappier ${ }^{3}$, ont violemment critiqué l'inaction et le retard du Québec sur le plan de la réglementation de la qualité de l'eau destinée à la consommation humaine (Brisson, 2000). Pour eux, le processus de modernisation passe par l'adoption d'une mesure de la transparence ou de la turbidité de l'eau. Pour Michèle Prévost : « le règlement actuel est si désuet qu'il met en danger la protection de la santé" (Lemieux, 1998). Les réactions de ces deux chercheurs ont été largement médiatisées. Devant cette situation, le ministre de l'Environnement de l'époque a pris l'engagement de combler ce retard.

\section{c. La proposition de 2000}

En 1998, la gestion de l'eau au Québec est de plus en plus questionnée. Afin d'obtenir un portrait de la situation, le ministère de l'Environnement demande au Bureau d'audience publique sur l'environnement (BAPE) d'investiguer et de tenir une audience publique. La première recommandation du rapport rendu public le 3 mai 2000 est d'adopter le plus rapidement possible de nouvelles normes et exigences de contrôle de l'eau destinée à la consommation humaine (Bureau d'audiences publiques sur l'environnement, 2000). Cet événement dans l'environnement interne du ministère de même que les échanges tenus lors du Congrès de Réseau environnement ont influencé le projet de règlement acheminé au Conseil des ministres du 18 mai 2000. Contrairement à la proposition de 1998, le nouveau projet prévoit adopter une norme concernant la turbidité (Gouvernement du Québec, 2000a), ce qui permettrait au Québec de se comparer aux autres provinces canadiennes et progressivement aux Etats-Unis. Ce projet demande des investissements progressifs de $430 \mathrm{M} \$$ et un montant supplémentaire de $80 \mathrm{M} \$, 10$ ans après l'adoption du règlement, pour adopter les normes

\footnotetext{
${ }^{2}$ La turbidité est un paramètre important pour contrôler la qualité de l'eau. Elle permet de mesurer l'aspect plus ou moins trouble de cette dernière.

${ }^{3}$ L'Institut Armand-Frappier et une composante de l'Institut national de la recherche scientifique (INRS). Ce centre de recherche regroupe une cinquantaine de professeurs-chercheurs spécialisés en immunologie et en microbiologie.
} 
américaines de turbidité et de trihalométhane (Gouvernement du Québec, 2000a). En plus de cet axe de révision, le projet prévoit également la mise à jour des normes en fonction des recommandations canadiennes, l'augmentation du nombre de réseaux assujettis, la désinfection des eaux de surface, l'augmentation de la fréquence de prélèvements et du nombre de paramètres analysés (Gouvernement du Québec, 2000a).

Le contenu de ce projet a été réévalué à la suite de la contamination par la bactérie E. coli de l'eau distribuée à Walkerton (Ontario) (Agence de santé publique du Canada, 2000). Ce malheureux événement de même que les nombreuses critiques adressées au gouvernement ontarien (Perkel, 2000) ont encouragé les décideurs du ministère de l'Environnement à prévoir des mesures particulières pour éviter une situation similaire au Québec. Le 9 juin 2000, quatre ajustements sont acheminés au Conseil des ministres. Ils abordent la formation obligatoire des opérateurs, le transfert électronique des données, la désinfection des eaux souterraines sous l'influence des eaux de surface et l'augmentation de la fréquence des prélèvements pour les réseaux de moins de 8000 personnes (Gouvernement du Québec, 2000b). Un autre mémoire complémentaire est envoyé au Conseil des ministres le 28 juin 2000. Les deux nouveaux ajustements étaient justifiés pour des raisons de santé publique. Le premier propose de rendre obligatoire l'échantillonnage pour tous les réseaux publics et privés et le second recommande l'entrée en vigueur du règlement 15 jours après sa publication (Gouvernement du Québec, 2000c).

À la fin du mois de juin, un changement dans le contexte externe favorise l'adoption de la nouvelle réglementation. Le gouvernement du Québec reçoit alors la confirmation que le gouvernement fédéral est prêt à investir $200 \mathrm{M} \$$ pour financer la mise à niveau des équipements de filtration. Cette annonce est particulièrement intéressante pour les exploitants qui pourront bénéficier d'un programme de subventions pour le renouvellement de leurs infrastructures (Francoeur, 2000).

Le 30 novembre 2000, le ministre de l'Environnement Paul Bégin, propose de nouvelles modifications pour répondre aux commentaires transmis par la population lors de la consultation publique et aux recommandations d'un groupe interministériel de travail (Talbot et Turgeon, 2007). Ces ajustements s'inspirent également de la nouvelle réglementation ontarienne adoptée en août 2000. Ils abordent les questions relatives à la certification des opérateurs, à la filtration des eaux de surface, à l'assujettissement des propriétaires de puits individuels et au suivi en continu du chlore résiduel et de la turbidité (Gouvernement du Québec, 2000d). Les différentes modifications proposées intègrent des exigences à différentes étapes de la production de l'eau destinée à la consommation humaine. L'importance d'effectuer ce changement stratégique est démontré en mai 2001 lorsque près de 7100 habitants de la ville de North Battelford et du village de Battleford (Saskatchewan) ont souffert d'une maladie diarrhéique à la suite d'une éclosion de cryptosporidiose d'origine hydrique (Agence de santé publique du Canada, 2001 ). C'est un problème au niveau du décanteur de l'usine de traitement qui est à l'origine de la baisse de l'efficacité et de 
l'augmentation par la même occasion du niveau de turbidité de l'eau (Agence de santé publique du Canada, 2001). Cette épidémie confirme aux décideurs l'importance de réglementer l'ensemble du processus de traitement et de distribution de l'eau afin de protéger correctement la santé de la population.

En janvier 2001, le Sierra Legal Defense Fund publie un rapport sur l'état de l'eau potable au Canada. Le premier constat de ce groupe écologiste est qu'il existe une grande disparité au niveau des normes et des exigences de contrôle dans les provinces et territoires. Afin de remédier à la situation, il propose au gouvernement canadien de légiférer pour mettre fin à cette gestion disparate (Francoeur, 2001a). Cette idée de développer une loi fédérale est alors reprise par les conservateurs fédéraux. Le nouveau ministre de l'Environnement du Québec, André Boisclair, s'oppose à ce que le gouvernement canadien intervienne dans les champs de compétences des provinces. Cet événement dans le contexte externe de l'organisme a favorisé l'adoption de la proposition de modification à l'étude (Francoeur, 2001b).

Le contenu du projet de règlement a finalement exclu les puits individuels et les petits réseaux de moins de 20 utilisateurs (Francoeur, 2001c). La nouvelle réglementation a été adoptée le 30 mai 2001 par le Conseil des ministres. Les coûts liés à cette modernisation sont évalués à 660 M\$ (Ministère de l'Environnement, 2001). Afin de favoriser le respect des nouvelles normes, les réseaux de distribution public vont pouvoir bénéficier de deux programmes de financement : le programme d'aide financière d'Infrastructures-Québec et Travaux d'infrastructures Canada-Québec 2000 (Ministère de l'Environnement, 2001).

Tableau 1 - Contenu et contexte du changement (1986-2001)

\begin{tabular}{|c|c|c|}
\hline Contenu & Contexte interne & Contexte externe \\
\hline Proposition de 1993 & & - Crise des finances publiques \\
\hline Proposition de 1998 & $\begin{array}{l}\text { - Mise en place d'une table } \\
\text { d'élaboration en } 1995 \\
\text { - Congrès annuel de Réseau } \\
\text { environnement (1998) }\end{array}$ & $\begin{array}{l}\text { - Crise des finances publiques } \\
\text { - Évolution de l'opinion publique }\end{array}$ \\
\hline $\begin{array}{l}\text { Proposition de } 2000 \\
\text { - Projet original (18 mai 2000) } \\
-1^{\text {ère }} \text { modification du projet } \\
\text { (9 juin 2000) } \\
-2^{\mathrm{e}} \text { modification du projet } \\
\text { (28 juin 2000) } \\
-3^{\mathrm{e}} \text { modification du projet } \\
\text { (30 novembre } 2000) \\
-4^{\mathrm{e}} \text { modification du projet } \\
\text { ( } 17 \text { mai } 2001) \\
\text { - Adoption du nouveau } \\
\text { règlement (30 mai 2001) }\end{array}$ & & $\begin{array}{l}\text { - La contamination de l'eau potable } \\
\text { à Walkerton (2000) } \\
\text { - Adoption d'un nouveau règlement } \\
\text { en Ontario (2000). } \\
\text { - Le gouvernement fédéral est prêt } \\
\text { à investir dans les équipements de } \\
\text { filtration (2000). } \\
\text { - La contamination de l'eau à } \\
\text { North Battleford (2001) } \\
\text { - Le gouvernement fédéral pense } \\
\text { à légiférer sur la réglementation } \\
\text { de l'eau potable (2001). } \\
\text { - Amélioration des finances publiques } \\
\text { du Québec à partir de 1998. }\end{array}$ \\
\hline
\end{tabular}


Le contenu et le contexte entourant ce changement stratégique ont rapidement évolué à la suite de la tenue du Congrès annuel de Réseau environnement en 1998. Certains événements du contexte extrerne, comme l'intention du gouvernement fédéral d'intervenir par une réglementation nationale ou encore la contamination de l'eau à Walkerton ont permis au Québec de se positionner parmi les États ayant les normes de qualité les plus élevées et des contrôles de production parmi les plus rigoureux sur le continent nord-américain. De plus, il ne faut pas sousestimer l'influence jouée par le contexte économique dans l'adoption de cette nouvelle réglementation. Les différents engagements politiques ont été favorisés par la participation financière du gouvernement fédéral et par une amélioration de l'état des finances publique. Le contenu du changement a été influencé par le contexte, mais il a également contribué à sa modification.

\section{Le processus de changement}

Le processus de changement réfère aux actions, réactions et interactions entre les différents acteurs intéressés par la proposition de changement (Pettigrew \& al., 1992). Au cours du processus de formulation de la nouvelle réglementation, deux acteurs du ministère de l'Environnement ont joué des rôles clés. II s'agit des deux ministres Paul Bégin (25 août 1997 au 8 mars 2001) et André Boisclair (8 mars 2001 au 29 avril 2003). Le premier est à l'origine de toute la réflexion gouvernementale sur la gestion de l'eau au Québec. C'est lui qui a entre autres mandaté le BAPE pour investiguer et réaliser une audience publique concernant la gestion de cette ressource naturelle. II a également pris comme engagement en 1998 et en 2000 de moderniser la réglementation de l'eau potable pour combler le retard du Québec. II a joué un rôle clé dans la recherche d'un compromis entre les impacts économiques et la protection de la santé publique et contribué à l'entente avec le fédéral en ce qui concerne un programme de financement des infrastructures (300 M\$). De plus, après Walkerton (2000), il a profité de l'appui de l'opinion publique et d'un contexte plus réceptif pour modifier le projet original (Pettigrew et al. 1992).

Pour sa part, André Boisclair a joué un rôle de médiateur afin de concilier les objectifs de protection de la santé publique et les impératifs économiques. À la toute fin du processus de formulation, il a accepté la demande des unions municipales d'exclure les réseaux desservant moins de 20 personnes. Cette décision a permis de favoriser l'adoption de la nouvelle réglementation. II ne faut pas oublier que les unions municipales sont à l'origine du retard du gouvernement à promulguer de nouvelles normes et contrôles dans les années 1990 (Bureau d'audience publique en environnement, 2000). II a mobilisé la majorité des organisations, à l'exception des exploitants publics et industriels, autour du projet de changement. La plupart ont reconnu la désuétude du règlement de 1984 et l'importance d'agir pour protéger la santé de la population. Cette collaboration a eu pour effet de rendre le contexte plus réceptif. Ces deux acteurs sont ce que Pettigrew et ses collaborateurs (1992) ont défini comme des personnes clés dans des positions critiques pour mener le changement. 
Pour étudier les relations entre les différents acteurs à l'extérieur de l'organisation, le concept de coalitions de cause a été mobilisé. Pour les besoins de l'analyse, les différentes parties prenantes ont été regroupées en 7 grandes catégories d'acteurs : les exploitants privés et industriels $(E P I=5)$, les exploitants publics $(E P=3)$, les chercheurs $(C=3)$, les ingénieurs $(I=1)$, les acteurs de santé publique $(S P=2)$, les spécialistes en environnement $(\mathrm{SEE}=$ 3 ) et finalement les laboratoires d'essais et microbiologistes $(L E M=2)$.

II est possible de dégager deux grandes coalitions (Figure 2). La première comprend les exploits privés et industriels (anti-réglementation) et la deuxième est composée des exploitants publics, des chercheurs, des acteurs de santé publique, des laboratoires d'essais, des microbiologistes et des spécialistes en environnement (pro-réglementation). En ce qui concerne les ingénieurs, il est impossible de les joindre à l'une ou l'autre de ces deux coalitions étant donné qu'ils n'ont pas tenté de coordonner leurs actions avec les autres catégories d'acteurs. Le positionnement des exploitants publics est surprenant si l'on prend en considération le rôle de blocage qu'ils ont joué avec les exploitants privés et industriels au cours des années 1990. En se basant sur la théorie des coalitions de cause (Sabatier et Jenkins-Smith, 1999 ; Sabatier et Weible, 2007) il est possible de croire que l'opposition des exploitants publics était basée sur des aspects secondaires comme le montant de la subvention plutôt que sur des valeurs ou des croyances plus fondamentales. Contrairement aux exploitants privés et industriels, ils ont reconnu dans les mémoires déposés lors de la consultation publique (2000) l'importance d'intervenir pour protéger la santé de la population. De plus, les exploitants privés et industriels, qui desservent majoritairement moins de 200 personnes, ne peuvent pas bénéficier d'un programme de financement des infrastructures comme les exploitants publics (Gouvernement du Québec, 2000a).

Figure 2 - Les coalitions

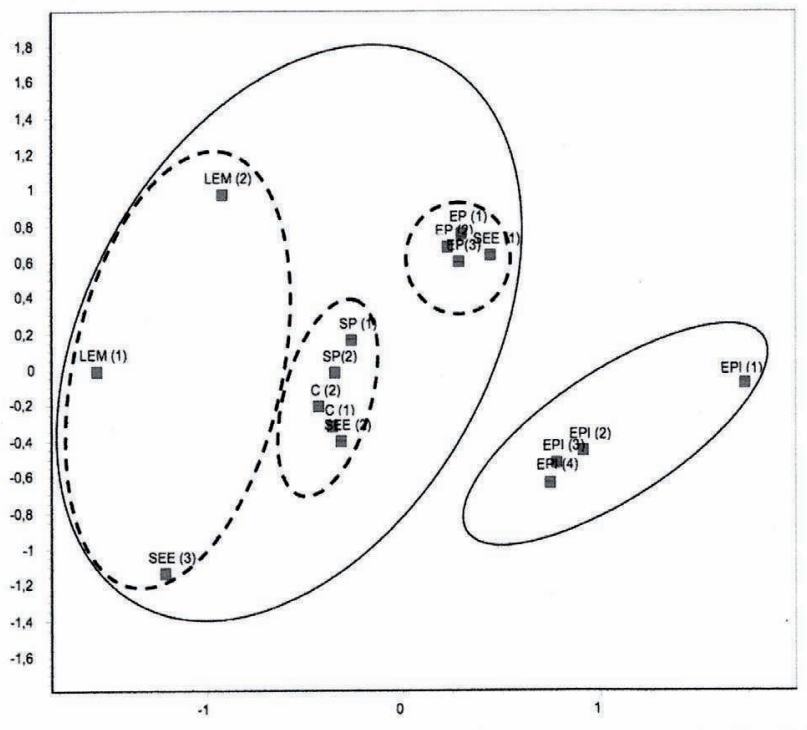


La coalition dominante (Sabatier et Jenkins-Smith, 1999 ; Sabatier et Weible, 2007) dans le cas à l'étude est le regroupement pro-réglementation. Cette dernière a commencé à avoir l'appui des décideurs publics après la tenue du Congrès annuel de Réseau environnement en 1998. Pour la majorité des membres de cette coalition, le processus de révision avait abouti, en 1997, à une proposition gouvernementale qui ne contenait pas les éléments essentiels des règlements sur la qualité de l'eau potable. Pour obtenir des modifications correspondant davantage à leurs valeurs, ils ont alerté la population et les autorités gouvernementales des dangers pour la santé liés à la consommation de l'eau potable. Certains chercheurs de cette coalition comme Pierre Payment et Michèle Prévost ont utilisé les résultats de leurs recherches pour démontrer que la majorité des stations de traitement de l'eau au Québec ne sont pas dotées d'équipements adéquats pour assurer l'enlèvement ou l'inactivation de certains micro-organismes pathogènes. Après les événements de Walketon (2000), cette coalition a bénéficié de l'appui de l'opinion publique (Sabatier et Weible, 2007).

Une autre ressource importante de ce regroupement d'acteurs est l'information scientifique (Sabatier et Weible, 2007). Comme on peut le voir dans la figure 2, les acteurs de santé publique et les chercheurs ont occupé une position centrale (Kriesi et Jegen, 2001) dans ce réseau d'acteurs. La deuxième coalition, les exploitants privés et industriels, a pour sa part perdu son pouvoir politique quand la situation financière du Québec s'est rétablie et que le gouvernement fédéral a avancé l'idée d'un nouveau programme pour financer les infrastructures. Après Walkerton (2000) et le rapport du Bureau d'audience publique en environnement (2000), un retour en arrière était impensable. Ces acteurs ne bénéficiant plus de l'appui des acteurs politiques, ont dû accepter certaines modernisations au règlement malgré les contraintes techniques et financières auxquelles ils faisaient face. De plus, le contexte entourant la prise de décision a évolué au milieu des années 1990 passant de conflictuel à collaboratif.

C'est au cours de cette période qu'est apparu le comité multisectoriel de réglementation de l'eau potable, au sein de Réseau environnement, regroupant des représentants de toutes les parties prenantes intéressées par cet enjeu. Cette organisation a joué un rôle central dans les négociations entourant la nouvelle réglementation en favorisant un rapprochement entre les deux coalitions. Cette recherche d'un compromis peut s'expliquer par le lien de dépendance entre les spécialistes en environnement et les exploitants privés et industriels. Les exploitants privés et industriels avaient tout intérêt à partager leurs ressources avec les spécialistes en environnement compte tenu des ressources informationnelles et de l'accès privilégié aux autorités légales dont ceux-ci disposaient. De leur côté, les spécialistes en environnement devaient tenter d'obtenir l'appui de tous les exploitants (publics, privés, industriels) pour influencer les décideurs et de cette façon obtenir une politique qui correspond à leurs croyances.

L'utilisation du concept de coalition a permis de bien comprendre l'interaction entre les notions de processus, de contenu et de contexte. De plus, la subdivision de la notion de processus en deux dimensions 
(interne et externe) a démontré sa pertinence. II est essentiel pour les parties prenantes d'avoir un accès privilégié aux acteurs politiques s'ils veulent faire entendre leurs arguments. Pour obtenir de bons résultats le contexte interne et externe au changement doit être réceptif. Par exemple, l'amélioration de l'état des finances publiques et la disponibilité des fonds fédéraux a permis aux acteurs de la coalition pro-réglementation d'obtenir une influence politique prépondérante auprès des acteurs politiques.

Conclusion
Cet article permet de mieux comprendre le processus ayant mené à l'adoption de ce changement stratégique. L'adoption de la nouvelle réglementation de l'eau potable au Québec a été influencée par le contexte et le jeu des acteurs à l'intérieur et à l'extérieur du ministère de l'Environnement. Trois grands facteurs contextuels ont favorisé l'adoption de ce changement. Premièrement, l'amélioration de la situation financière a permis d'adopter des normes et des contrôles très rigoureux. La nature et les orientations du changement ont été influencées par le redressement des finances publiques et la disponibilité d'un financement fédéral. Deuxièmement, les contaminations de l'eau à Walkerton (2000) et à North Battleford (2001) ont sensibilisé la population, l'opinion publique et les autorités gouvernementales aux dangers liés au maintien de normes désuètes. Ces événements ont permis d'accélérer l'adoption d'une nouvelle réglementation et d'en modifier les termes. Finalement, le contexte politique a évolué après la nomination d'un nouveau ministre de l'Environnement et la menace de voir le gouvernement fédéral intervenir. Tous ces facteurs contextuels ont rendu l'environnement entourant le changement plus réceptif. La coalition proréglementation a profité de la situation pour dominer les débats entourant la formulation de cette politique dès 1998. Ses ressources informationnelles et politiques lui ont permis d'être entendu par les acteurs politiques et d'obtenir une réglementation correspondant à ses valeurs. Ce changement n'aurait pu être possible sans l'action des acteurs politiques en place. Ils ont favorisé les compromis pour profiter de la fenêtre d'opportunité créée grâce à une évolution de l'opinion publique, de la situation économique et politique.

L'utilisation de l'approche contextualiste s'est avéré un choix judicieux pour "reconstruire » le processus ayant mené à la décision de réglementer cette ressource naturelle par les autorités gouvernementales. Cette étude a trois principaux apports au niveau de la recherche sur le changement. Premièrement, elle démontre l'utilité du contextualisme pour l'étude de l'adoption d'une nouvelle mesure gouvernementale. Deuxièmement, elle propose de scinder la notion de processus de l'approche contextualiste en deux dimensions soit l'interne et l'externe en faisant appel à la notion de coalitions de cause (Sabatier et Jenkins-Smith, 1999) pour y étudier les interactions entre les différentes parties prenantes. Troisièmement, il s'agit de l'une des rares recherches à avoir eu recours à l'analyse structurale des réseaux sociaux pour l'analyse des relations de pouvoirs. L'utilisation de ce type d'analyse permet d'opérationnaliser la notion de réseau de politique, et ainsi, dépasser la simplicité souvent reprochée à cette notion (Macdonald, 2001). Pour les recherches futures, il serait intéressant de mobiliser ces 
méthodes d'analyse pour examiner le jeu des acteurs à l'intérieur de l'organisation. De plus, des recherches supplémentaires devront aborder la notion de réceptivité du contexte (Pettigrew et al., 1992) afin de mieux cerner son influence sur les regroupements et les actions des différentes parties prenantes.

Cette étude a également certaines limites. Premièrement, il aurait été intéressant d'avoir plusieurs mesures dans le temps afin d'évaluer les relations entre les acteurs. Certains éléments contextuels ont pu influencer les données utilisées dans le cadre de cette étude. II suffit de penser au positionnement des exploitants publics qui a pu évoluer depuis 2001. II faut cependant relativiser cette critique puisque selon Sabatier et Jenkins-Smith (1993; 1999) les coalitions sont stables pendant plus d'une décennie et que la nouvelle coalition dominante occupe cette position depuis maintenant un peu plus de 10 ans. Deuxièmement, afin de mieux décrire le processus de transformation, il aurait été approprié d'interroger les politiques qui ont participé aux discussions entourant cette transformation. Ainsi, il aurait été possible de mieux comprendre l'importance qu'ils ont accordée à certains événements de même qu'aux actions politiques des différents acteurs.

\section{BIBLIOGRAPHIE}

Adam S. \& Kriesi H. M. (2007), "The Network Approach». dans P. A. Sabatier (Ed.), Theories of the Policy Process, 2 ed. San Francisco, Westview Press. p. 189-220.

Agence de santé publique du Canada. (2000), «Éclosion de gastro-entérite d'origine hydrique associée à un réseau d'aqueduc municipal contaminé ", Relevé des maladies transmissibles au Canada, vol. 26, no 20, p. 170173.

Agence de santé publique du Canada. (2001), «Éclosion de cryptosporidiose d'origine hydrique, North Battleford ». Relevé des maladies transmissibles au Canada, vol. 27, no 22, p. 185-192.

Brisson B. (2000), "Eau potable : la précipitation après des années d'inaction », La Presse, p. A3.

Bureau d'audiences publiques sur l'environnement. (2000), L'eau, ressource à protéger, à partager et à mettre en valeur, Bureau d'audiences publiques sur l'environnement.

Caza A. (2000), « Context Receptivity: Innovation in an Amateur Sport Organization ", Journal of Sport Management, no 14, p. 227-242.

Charland G. (2006), "Le Québec comparé et les finances publiques au Canada, 1992-2002 » dans J. Crête (Ed.), Politiques publiques : le Québec comparé, Saint-Nicolas, Les Presses de l'Université Laval.

Cho S., Mathiassen L. \& Nilsson A. (2008), «Contextual dynamics during health information systems implementation: an event-based actor-network approach ", European Journal of Information Systems, vol. 17, no 6, p.614-630. 
Craun M. F. (2006), «Waterborne outbreaks reported in the United States », Journal of Water and Health, no 4, p. 19-30.

Demers C. (2007), Organizational Change Theories, Sage Publication.

Dowding K. (2001), «There Must Be End to Confusion: Policy Networks, Intellectual Fatigue, and the Need for Political Science Methods Courses in British Universities ». Political Studies, vol. 49, no 1, p. 89-105.

Francoeur L.-G. (2000), «Ottawa est prêt à fournir 200 millions au Québec », Le Devoir, p. A8.

Francoeur L.-G. (2001a), «Le fédéralisme des cancers », Le Devoir, p. A1.

Francoeur L.-G. (2001b), «Eau potable: Québec attend pour mieux frapper », Le Devoir, p. A3.

Francoeur L.-G. (2001c), «Règlement sur l'eau : Boisclair exclu les petits réseaux », Le Devoir, p. A2.

Girginov V. \& Sandanski I. (2008), «Understanding the Changing Nature of Sports Organisations in Transforming Societies", Sport Management Review, vol. 11, no 1, p. 21-50.

Gouvernement du Québec. (1993), Mémoire au conseil des ministres: Modification au Règlement sur l'eau potable, Gouvernement du Québec.

Gouvernement du Québec. (2000a), Mémoire au Conseil des ministres. Projet de modification du Règlement sur l'eau potable, Gouvernement du Québec.

Gouvernement du Québec. (2000b), Mémoire complémentaire au Conseil des ministres. Projet de modification du Règlement sur l'eau potable, Gouvernement du Québec.

Gouvernement du Québec. (2000c), Mémoire complémentaire au Conseil des ministres. Projet de modification du Règlement sur l'eau potable, Gouvernement du Québec.

Gouvernement du Québec. (2000d), Mémoire au Conseil des ministres. Projet de modification du Règlement sur l'eau potable, Gouvernement du Québec.

Hrudey S. E., Payment P., Huck P. M., Gillham R. W. \& Hrudey E. J. (2003). «A fatal waterborne disease epidemic in Walkerton, Ontario : comparison with other waterborne outbreaks in the developed world », Water Science and Technology, vol. 47, no 3, p. 7-14.

Jayasuriya R. (1999). «Managing information systems for health services in a developing country: a case study using a contextualist framework ", International Journal of Information Management, vol. 19, no 5, p. 335-349.

John P. (2003), «Is there life after policy streams, advocacy coalitions, and punctuations : using evolutionary theory to explain policy change ", Policy Studies Journal, vol. 31, no 4, p. 481-498.

Knoke D., Broadbent F. U. \& Tsujinaka, Y. (1996), Comparing Policy networks. Labour Politics in the US, Germany and Japan, Cambridge, Cambridge University Press. 
Kriesi H. \& Jegen, M. (2001), «The Swiss energy policy elite: The actor constellation of a policy domain in transition », European Journal of Political Research, vol. 39, no 2, p. 251-287.

Kriesi H., Adam S. \& Jochum, M. (2006), «Comparative analysis of policy networks in Western Europe », Journal of European Public Policy, vol. 13, no 3, p. 341-361.

Kübler D. \& Maillard J. (2009), Analyser les politiques publiques, Grenoble, PUG.

Laumann E. O. \& Pappi F. U. (1976). Networks of Collective Action: A Perspective on Community Influence Systems, New York, Academic Press.

Laumann E. O. \& Knoke D. (1987). The Organizational State. Social Choice in National Policy Domains, Madison, The University of Wisconsin Press.

Leclerc H. (2003), " $Y$ a-t-il des infections bactériennes opportunistes transmises par les eaux d'alimentation? », Journal Européen d'Hydrologie, vol. 34, no 1, p. 11-44.

Lemieux L. (1998), «Eau: les normes québécoises en retard de 20 ans », Le Soleil, p. A11.

Levallois P. (2006), «Eau potable et santé publique : défies actuels et futures ", Revue des Sciences de l'Eau, vol. 19, no 2, p. 127-135.

Macdonal D. (2001), "Coerciveness and the selection of environmental policy instruments ", Canadian Public Administration, vol. 44, no 2, p. 161-187.

Marchionni C. \& Ritchie J. (2008), « Organizational factors that support the implementation of a nursing Best Practice Guideline ", Journal of Nursing Management, vol. 16, no 3, p. 266-274.

Ministère de l'Environnement. (2001), Qualité de l'eau potable : Québec se donne les plus hauts standards en Amérique du Nord. Ministère de l'Environnement.

Ministère de l'Environnement du Québec. (1989). L'eau potable au Québec un premier bilan de sa qualité, Ministère de l'Environnement du Québec.

Perkel C. (2000). «Queen's Park révise ses règles sur la gestion de l'eau potable ", Le Devoir, 30 mai 2000, p. A1.

Pettigrew A. M. (1990), «Longitudinal field research on change: theory and practice », Organization Science, vol. 1, no 3, p. 267-292.

Pettigrew A. M., Ferlie E. \& McKee L. (1992). Shaping strategic change. Making change in large organizations. The case of the National Health Service, London, Sage Publications.

Pettigrew A. M., Woodman R. W. \& Cameron K. S. (2001), « Studying organizational change and development: Challenges for future research ", Academy of Management Journal, vol. 44, no 4, p. 697-713.

Sabatier P. A. \& Jenkins-Smith H. C. (1993), Policy change and learning. An advocacy coalition approach, San Francisco, Westivew Press. 
Sabatier P. A. \& Jenkins-Smith H. C. (1999), « The Advocacy Coalition Framework. An Assessment ", dans P. A. Sabatier (Ed.), Theories of the Policy Process, San Francisco: Westview Press, p. 117-166.

Sabatier P. A. \& Weible C. M. (2007), « The Adovocacy Coalition Framework. Innovations and Clarifications ", dans P. A. Sabatier (Ed.), Theories of the Policy Process, San Francisco, Westview Press, p. 189-220.

Schoenen D. (2002), « Role of disinfection in suppressing the spread of pathogens with drinking water : possibilities and limitations ", Water Reasearch, no 36, p. 3874-3888.

Schuster C. J., Ellis A. G., Robertson W. J., Charron D. F., Aramini J. J., Marshall B. J. \& Medeiros D. T. (2005), «Infectious Disease Outbreaks Related to Drinking Water in Canada, 1974-2001 », Canadian Journal of Public Health, vol. 96, no 4, p. 254-258.

Skirstad B. (2009), « Gender policy and organizational change: A contextual approach », Sport Management Review, vol. 12, no 4, p. 202-216.

Starkey K. P. (1987). «Book review and response », Journal of Management Studies, vol. 24, no 4, p. 413-426.

Talbot D. \& Turgeon J. (2007), Le Règlement sur la qualité de l'eau potable: étude de cas, Québec, Groupe d'études sur les politiques publiques et la santé, p. 11.

Thibault L. \& Babiak K. (2005), « Organizational Changes in Canada's Sport System: Toward an Athlete-Centred Approach », European Sport Management Quarterly, vol. 5, no 2, p. 105 - 132.

Weible C. W. \& Sabatier P. A. (2005), « Comparing Policy Networks: Marine Protected Areas in California », Policy Studies Journal, vol. 33, no 2, p. 181-201.

Yin R. K. (1994), Case study research Design and Methods, London, Sage publication. 
